\title{
Design and Implementation of Students' Information Management System (SIMS) Based on ASP
}

\author{
Cheng-Hui YANG ${ }^{1,2, a, *}$ \\ ${ }^{1}$ College of Electrical Engineering, Northwest University for Nationalities, Lanzhou, Gansu, China \\ ${ }^{2}$ School of Automation \& Electrical Engineering, Lanzhou Jiaotong University, China \\ ayangchenghui36@163.com \\ ${ }^{*}$ Corresponding author
}

Keywords: ASP, B/S Structure, Student Information Management System, Microsoft Office Access

\begin{abstract}
The graduation project is based on B/S three-tier management system, which uses ASP technology and takes the main script language by VbScript. What system's database use is Microsoft Office Access. System consists of system management, school register management, performance management, account management, data management and personal management of six modules. In the paper first briefly elaborates system's research background and the research goal and the significance, introduces some technology and the theory of knowledge that relevant of system development. Then introduces the management system design and implementation process, which including the design of backstage supporter's database, system' homepage design, etc. Finally carries on the test to the system, then summarize the questions and the solution method that encountered in system development process.
\end{abstract}

\section{Introduction}

With the computer, multimedia and network technology development, human entered the information age. People using these advanced technologies can be more convenient and efficient reuse of resources, rapid exchange of information. Traditional static Web site development and maintenance become increasingly difficult, while growing and changing information, so that site maintenance personnel often have to modify their pages, especially based on database-driven Web site even more so, other On the one hand because it is not static pages for effective interaction with the viewer, so that people feel more and more boring, rather than once again into the same site. Therefore, development of dynamic web pages or dynamic content has become more and more sites to pursue goals.

Student information management system should be able to provide users with sufficient information and fast query tool. But there has been the way people use the traditional manual management of paper files, this management approach, there are many shortcomings, such as: low efficiency, poor security, the other over time, will produce a large number of files and data, which find, update and maintain have brought a lot of difficulties. Therefore, the students of computer information processing, networking, school management is to achieve modernization and an important part of information technology.

\section{Software and L anguage Description}

\section{M icr osoft Office Access I ntroduction}

Microsoft Office Access(Formerly Microsoft Access)is published by Microsoft relational database management system. It combines the Microsoft Jet Database Engine, and two graphical user interface features, is a member of Microsoft Office.

It has the following six advantages:

(1) storage is simple, easy maintenance and management. 
(2) Object-oriented Access is an object-oriented development tool, object-oriented approach to database systems in a variety of functional objects; it will be a variety of database management functions encapsulated in various types of objects.

(3) User-friendly, easy to operate.

(4) Integrated environment, handle a variety of data.

(5) Access supports ODBC (development of database connectivity, Open Data Base Connectivity), the use of Access Powerful DDE (Dynamic Data Exchange) and OLE (object link and embedding) features in a data table, embedded bitmaps, sounds, Excel spreadsheet, Word documents, you can create dynamic database reports and forms and so on.

(6) Supports a wide range, easy to expand, greater flexibility.

\section{ASP Overview}

Active Server Page ASP is the acronym meaning "Active Server Pages." ASP is Microsoft's development of the CGI script instead of an application, it can interact with databases and other programs, is a simple and convenient programming tools.

ASP's implementation has the following steps [2]:

(1) User in the browser's address bar, enters the ASP file, and presses the Enter key to trigger the ASP request.

(2) Browser will send the request to the ASP Web server.

(3) Web server accepts the request and in accordance with. Asp ASP to determine the extension request.

(4) Web server from the hard drive or memory to read the correct ASP file.

(5) Web server will send the file to a specific file asp.dll.

(6) ASP file and execute from start to finish under the command will generate the required HTML file

(7) HTML files are transferred back to the browser.

(8) The user's browser interprets the HTML file and display the results.

\section{VbScript Language Description}

VBScript is short for Visual Basic Script, Visual Basic scripting language that is sometimes abbreviated as VBS. Is the default ASP dynamic web programming language, built-in objects with ASP and ADO objects, users will quickly master the dynamic ASP pages to access the database development technologies. In addition it also has the characteristics of the original language easy to learn. Currently this language is widely used in Web pages and ASP program production, but also can be used directly as an executable program. Simple VB statements for debugging very convenient.

\section{B / S Structure Introduced}

The so-called B / S (Browser / Server) structure that the browser / server model structure. WEB is the rise of a network model, WEB browser is the client the most important applications. This model unifies the client, the system functions to achieve the core of the focus to the server to simplify system development, maintenance and use. Just install the client on a browser (Browser), such as Internet Explorer, Microsoft Office Access server installation or SQL Server and other databases. Web Server via a browser will be able to exchange data with the database.

\section{System Design}

\section{System F unctions Into}

The system consists of system management, school management, performance management, account management, data management and personal management of six modules, users access the system by logging the browser.

Users can be divided into two categories: 
First, Manage User is responsible for student information to add, delete, modify, query and other operations, such users have a higher authority, they can also maintain the system.

Secondly, the student user, such user's operating authority is relatively low, they can only query their own information, if you encounter an error message the problem can be feedback to the administrator.

\section{Database Design}

The system uses Microsoft Office Access a database SIMS.mdb, including the following eight tables:

(1) Users of the system administrator to save table SIMS_Admin relevant information.

(2) Database used to store backup information table SIMS_Data database of information.

(3) The system administrator to save the log table SIMS_Log operates in the background information.

(4) Student achievement table SIMS_Score to hold individual students performance at school.

(5) Students' personal information is used to save the table SIMS_Stu students in school-related information.

(6) Student status information table SIMS_Info to save the time of enrollment enrollment information.

(7) Student Curriculum SIMS_Pro used to save information about individual students, curriculum.

(8) Students to save personal information table SIMS_Stu students in school-related information.

(9) Lookup table SIMS_Search student achievement is a select query relational table.

\section{System Implementation}

\section{Configuration Database}

Build the database, a new ASP file conn.asp, its implementation is very simple code to connect to the database, the concrete realization of the following code.

$<\%$

Dim conn

set conn $=$ server.createobject("adodb.connection")

conn.connectionstring="Provider=Microsoft.Jet.OLEDB.4.0,DataSource="

\&Server.MapPath("data/db.mdb")

conn.open

$\%>$

\section{Admin_login.Asp Login Screen Background}

All users back into the student information management system, you must enter a user name, password and verification code, and then to the data table SIMS_Admin query if the user exists, if there is to judge the user entered password is correct, true if the password continued to determine whether the verification code consistent with the prompt, the user is some information written in the ASP Session object built after the procedure calls for the final jump into the Admin Home page, otherwise prompt the user password.

\section{Manage Home Design}

Home main advantage of the background framework <FRAMESET > </ FRAMESET > With $<$ FRAME > </ FRAME > To the design, implementation, the head page (admin_header.asp), the menu page (admin_menu.asp), the main content pages (admin_main.asp) on the same page shows that the specific code is shown below.

<FRAMESET border=0 frameSpacing=0 rows="60, * frameBorder=0>

$<$ FRAME name=admin_header src="admin_header.asp" frameBorder=0 noResize scrolling=no > $<$ FRAMESET cols="170, *"> 
<FRAME name=admin_menu src="admin_menu.asp" frameBorder=0 noResize>

<FRAME name=admin_main src="admin_main.asp" frameBorder=0 noResize scrolling=yes >

$</$ FRAMESET $>$

$</$ FRAMESET $>$

$<$ noframes $>$

$</$ noframes $>$

\section{Function M odules}

System management, school management, performance management, account management, data management and personal management of six modules can page through the appropriate action, execute SQL commands on the database SIMS.mdb, in order to achieve the appropriate module functions. For example, students can enter the student number P071613598 to check the student performance at school, student information, etc.

\section{System Testing}

Tem testing is intended to ensure that the implementation of the system is indeed the user want. To achieve this, then take a series of test events.

The design of the system has been tested and browser test page to ensure the system is running without an error page. After several modifications and debugging, the system most of functions are to be achieved, but there are some features not yet perfect, it remains to be further developed.

\section{Conclusions}

Current college students in the study of information management problems and improve, based on the use of technology and VbScript ASP programming language to implement the Student Information Management System Design and Implementation.

By using the Student Information Management System administrators can accurately to student information into the database, student information at any time to query, modification and other operations. Thus greatly reducing the teaching workload of staff input student information, to enable students to systematic management, standardization, automation, thereby enhancing the efficiency of information management students.

\section{Acknowledgement}

This work was supported by National Natural Science Foundation of China. (Grant NO. 61263002) and by Ministry of Science and Technology of China under National 973 Basic Research Program (Grant 2011CB302805).

The project also supported by Educational reform research Funds of Northwest University for Nationalities, Northwest University for Nationalities, China. (Grant NO. JG201135). And the project also supported by two class excellent courses research Funds of Northwest University for Nationalities, Northwest University for Nationalities, China. ( Grant NO. JPKC201105, Grant NO. JPKC201136).

\section{References}

[1] Pang Yajuan, Sun Mingli, Lvji Di. ASP network programming self-study manual. Beijing: People's Posts and Telecommunications Press, 2008.3 (2009.7 reprint).

[2] Han Dianyuan. ASP dynamic website development and application of technology, Nanjing: Nanjing University Press, 2007.4.

[3] Shi Zhiguo, Wang Zhiliang, Xue people. ASP tutorial case Jing Xie, Beijing: Tsinghua 
University Press, Northern Jiaotong University Press, 2004.1.

[4] Dou Wanfeng, etc. Software Engineering Methods and Practice, Beijing: Mechanical Industry Press, 2009.5.

[5] Li Ying. ASP + SQL Server 2005 project development from entry to the master. Beijing: People's Posts and Telecommunications Press, 2007.9.

[6] Zhang Xinwei, web pages classic color art. Beijing: Electronic Industry Press, 2009.6.

[7] (U.S.) Dusan Petkovic. Feng, Xue Ying translation. Database system and SQL Server [M]. Microsoft SQL Server 2005 Beginners Guide Beijing edition: Tsinghua University Press, 2007.4

[8] Li Mufang. Dreamweaver CS4 + ASP dynamic website development from basic to practice. Beijing: Mechanical Industry Press, 2010.03.

[9] Li Suruo, Yan Yongsong, Chen Wanhua. ASP dynamic website design. Beijing: Chemical Industry Press, 2010.01.

[10] Tomorrow Technology ed. ASP development typical module Daquan. Beijing: People's Posts \& Telecom Press, 2009.04. 